\title{
Scalar field dark matter and pulsar timing observations
}

\section{Andrei Khmelnitsky*}

Ben-Gurion University of the Negev, Beer-Sheva, Israel

E-mail: andreykhepost.bgu.ac.il

\section{Valery Rubakov}

Institute for Nuclear Research of the Russian Academy of Sciences, Moscow, Russia

\begin{abstract}
An ultralight free scalar field with mass around $10^{-23}-10^{-22} \mathrm{eV}$ is a viable dark mater candidate, which can help to resolve some of the issues of the cold dark matter on sub-galactic scales. We consider the gravitational field of the galactic halo composed out of such dark matter. The scalar field has oscillating in time pressure, which induces oscillations of gravitational potential with amplitude of the order of $10^{-15}$ and frequency in the nanohertz range. This frequency is in the range of pulsar timing array observations. We estimate the magnitude of the pulse arrival time residuals induced by the oscillating gravitational potential. We find that for a range of dark matter masses, the scalar field dark matter signal is comparable to the stochastic gravitational wave signal and can be detected by the planned SKA pulsar timing array experiment.
\end{abstract}

Frontiers of Fundamental Physics 14

15-18 July 2014

Aix Marseille University (AMU) Saint-Charles Campus, Marseille, France

\footnotetext{
${ }^{*}$ Speaker.
} 


\section{Introduction and summary}

The standard $\Lambda \mathrm{CDM}$ cosmological model is very successful in describing properties of our Universe on large scales. However, there is a persisting mismatch between the observed properties of the structures on sub-galactic scales and the results of the structure formation simulations in cold dark matter (see e.g. [1] for a review). All known problems of CDM are different faces of the fact that the structures in CDM model are overabundant on sub-galactic scales. It is believed that these issues may find their explanation in complex astrophysical phenomena, which are not yet properly taken into account in the CDM simulations. Nevertheless, they provide a motivation to study dark matter models, in which the structure formation on sub-galactic scales is suppressed in comparison with the CDM, so-called warm dark matter models, see e.g. [2].

In this paper we consider an ultralight free scalar field dark matter. As discussed, e.g., in [3] (who build upon earlier works), a scalar field of mass $m=10^{-23}-10^{-22} \mathrm{eV}$ can serve as warm dark matter. This dark matter candidate is discussed in a number of papers, see e.g. [4] for a recent review and references. The main astrophysical consequence of this dark matter candidate is the suppression of the structures on the sub-galactic scales. In particular, one expects the dark matter density profiles to have smooth central cores, and the number of dwarf galaxies inside large galactic haloes to be considerably reduced in comparison to the CDM case. There are two main effects leading to such a suppression. First, the scalar field has a non-vanishing Jeans scale. This leads to a cutoff in the linear power spectrum of density perturbations at large wavenumbers [3]. Second, there is an effective quantum pressure resulting from the Heisenberg uncertainty principle. It smoothes out inhomogeneities in the dark matter distribution on scales smaller than the de Broglie wavelength $\lambda_{d B}$ of dark matter particles. Since the characteristic momentum of the dark matter particles is $k=m v$, where $v \approx 10^{-3}$ is the typical velocity in the Galaxy, one has

$$
\lambda_{d B}=\frac{2 \pi}{k} \equiv \frac{2 \pi}{m v} \approx 3.8 \mathrm{kpc}\left(\frac{10^{-23} \mathrm{eV}}{m}\right)\left(\frac{10^{-3}}{v}\right) .
$$

Therefore, the dark matter density distribution in the galactic halo is expected to be smooth on scales shorter than a few kiloparsec. We would also like to note, that an even lighter scalar field would behave as hot dark matter and would fail to form observed structures, unless it constitutes a subdominant fraction of all dark matter [5]. A heavier scalar field is a legitimate cold dark matter candidate.

Ultra-light non-interacting dark matter inside galactic halos is well described by a classical scalar field that oscillates with frequency $m$. It behaves as perfect fluid with oscillating pressure, which averages to zero on the time scales greater than the oscillation period, and is usually treated as a pressureless dust. However, it seems to be overlooked that the oscillations in pressure induce oscillations in gravitational potentials, which can, in principle, be observable.

We study the effect of such oscillations on photon arrival time and show that it can be detected using the next generation of pulsar timing array observations [6]. In particular, we find that the effect on the pulsar timing is comparable to the effect of a monochromatic gravitational wave with characteristic strain $h_{c} \approx 2 \cdot 10^{-15}\left(\frac{10^{-23} \mathrm{eV}}{m}\right)^{2}$, and frequency $f=5 \cdot 10^{-9} \mathrm{~Hz}\left(\frac{m}{10^{-23} \mathrm{eV}}\right)$. A signal of similar magnitude is expected from the stochastic gravitational wave background produced by massive black hole binary systems [7]. However, unlike the latter, the signal we consider is 
monochromatic. Such a signal will be in the reach of pulsar timing measurements based on the SKA observations [6].

\section{Time-dependent gravitational potential}

Taking into account that the number density of dark matter particles in the Galaxy is given by $n=\rho_{D M} / m$, we estimate the characteristic occupation number of dark matter in the galactic halo as

$$
\frac{\Delta N}{\Delta x^{3} \Delta p^{3}} \sim n / k^{3}=\frac{\rho_{D M}}{m k^{3}} \approx 10^{96}\left(\frac{\rho_{D M}}{0.3 \mathrm{GeV} / \mathrm{cm}^{3}}\right)\left(\frac{10^{-23} \mathrm{eV}}{m}\right)^{4} .
$$

Since the occupation number is huge, the dark matter in the Galaxy is in the domain of validity of the classical field theory, and can be described by a classical scalar field $\phi(\mathbf{x}, t)$. Generally, such a field can be represented as a collection of plane waves of typical momentum $k$. The frequencies are given by the corresponding energy, which in the non-relativistic limit equals $E \simeq m+m v^{2} / 2$. Since the characteristic time scale corresponding to the second term $(\Delta \omega)^{-1}=2 /\left(m v^{2}\right)$ is very large (the corresponding length scale is of order $1 \mathrm{Mpc}$ ), to the leading order we can set the frequency to be equal to $m$. Thus the field we are dealing with has the form

$$
\phi(\mathbf{x}, t)=A(\mathbf{x}) \cos (m t+\alpha(\mathbf{x})) .
$$

The gravitational field produced by the scalar field dark matter in the galactic halo is found in [8]. In the Newtonian gauge, in which the metric takes the following form:

$$
d s^{2}=(1+2 \Phi(\mathbf{x}, t)) d t^{2}-(1-2 \Psi(\mathbf{x}, t)) \delta_{i j} d x^{i} d x^{j},
$$

the relevant oscillating component has an amplitude $\Psi_{c}$ :

$$
\Psi_{c}(\mathbf{x})=\frac{1}{2} \pi G A(\mathbf{x})^{2}=\pi \frac{G \rho_{D M}(\mathbf{x})}{m^{2}},
$$

where the leading oscillating contribution is defined as:

$$
\Psi(\mathbf{x}, t) \simeq \Psi_{0}(\mathbf{x})+\Psi_{c}(\mathbf{x}) \cos (\omega t+2 \alpha(\mathbf{x})) .
$$

Note that the time-independent part is of order $\Psi_{0} \sim G \rho_{D M} / k^{2}$, and the oscillating part is indeed smaller than $\Psi_{0}$ by a factor of $k^{2} / \mathrm{m}^{2}=v^{2}$.

It is also possible, although less straightforward, to find the oscillating part of the potential $\Phi$. However, as we will see below, it does not contribute to the final result.

\section{Effect on the pulsar timing}

The time-dependent oscillations (2.5) of the metric induce a time-dependent frequency shift and a time delay for any propagating signal. Such a delay, in principle, can be captured in the pulsar timing experiments. For this one detects the pulse arrival times for a set of nearby millisecond pulsars, for which these times in the laboratory frame can be well modelled. The main effects that contribute to the variation of signal arrival times are the motion of the laboratory frame with respect 
to the Solar System barycentre, the peculiar motion of the pulsar, the dispersion by the interstellar medium, and the intrinsic variation of the pulsar period (cf. [9]). After all these effects are taken into account, the residual variation of the arrival times can be attributed to the effect of propagation in a time-dependent metric. In particular, this method is employed for gravitational wave search (see, e.g., [10] and references therein).

The time-dependent part of the timing residuals induced by the time variation (2.5) of the metric in the case when the ultralight scalar field is the major component of the galactic dark matter halo can be estimated as:

$$
\Delta t(t)=\frac{2 \Psi_{c}}{\omega} \sin \left(\frac{\omega D}{2}+\alpha(\mathbf{x})-\alpha\left(\mathbf{x}_{\mathbf{p}}\right)\right) \cos \left(\omega t+\alpha(\mathbf{x})+\alpha\left(\mathbf{x}_{\mathbf{p}}\right)-\frac{\omega D}{2}\right) .
$$

This time residual is oscillating in time with frequency $\omega$ and has the amplitude

$$
\Delta t_{D M}=\frac{2 \Psi_{c}}{\omega} \sin \left(\frac{\omega D}{2}+\alpha(\mathbf{x})-\alpha\left(\mathbf{x}_{\mathbf{p}}\right)\right) .
$$

It depends on the distance to the pulsar and the scalar field phase $\alpha$ at the locations of the pulsar and the detector.

The sensitivity of the pulsar timing array observations to the dark matter signal can be estimated by comparing (3.2) with the time-dependent timing residual induced by gravitational wave background. In the main paper [8] we show that the scalar field dark matter has the same effect on the pulsar timing measurements as gravitational wave background with characteristic strain

$$
h_{c}=2 \sqrt{3} \Psi_{c}=2 \cdot 10^{-15}\left(\frac{\rho_{D M}}{0.3 \mathrm{GeV} / \mathrm{cm}^{3}}\right)\left(\frac{10^{-23} \mathrm{eV}}{m}\right)^{2},
$$

at frequency

$$
f \equiv 2 \pi \omega=5 \cdot 10^{-9} \mathrm{~Hz}\left(\frac{m}{10^{-23} \mathrm{eV}}\right) .
$$

The amplitude of the signal from the scalar field dark matter for a range of masses $m$ is shown in Fig. 1 together with the sensitivity curves of the pulsar timing array experiments. The sensitivities are taken from [7] where three cases are considered. The current limit from the Parkes PTA [10] corresponds to $h_{c} \approx 2 \cdot 10^{-14}$ at the frequency $f=8 \cdot 10^{-9} \mathrm{~Hz}$. The sensitivity achievable by PPTA by monitoring 20 pulsars for 5 years with the timing precision $\delta t_{r m s}=100 \mathrm{~ns}$ is estimated as $h_{c} \approx 2 \cdot 10^{-15}$ at the frequency $f=7 \cdot 10^{-9} \mathrm{~Hz}$. Finally, assuming that SKA will be able to monitor 100 pulsars for 10 years with the timing precision $50 \mathrm{~ns}$, the sensitivity of $h_{c} \approx 10^{-16}$ at the frequency $f=3 \cdot 10^{-9} \mathrm{~Hz}$ can be achieved. We see from Fig. 1 that the scalar field dark matter signal can be observed with SKA pulsar timing array for the dark matter mass $m \lesssim 2.3 \cdot 10^{-23} \mathrm{eV}$.

We notice that while the signal from scalar field dark matter grows as the mass $m$ decreases, dark matter with $m<10^{-23} \mathrm{eV}$ would wash out structures on the observed scales and thus would behave as hot dark matter (cf. [3] and [11]). Hence, the interesting range of masses where the signal is detectable is fairly narrow, $10^{-23} \mathrm{eV}<m \lesssim 2.3 \cdot 10^{-23} \mathrm{eV}$.

To conclude, the pulsar timing signal from the scalar field dark matter is comparable to the signal expected from the stochastic gravitational wave background produced by the cosmic population of massive black hole binary systems [7]. It can be detected by the pulsar timing measurements at the planned SKA telescope. The scalar field dark matter signal has two signatures that distinguish it 


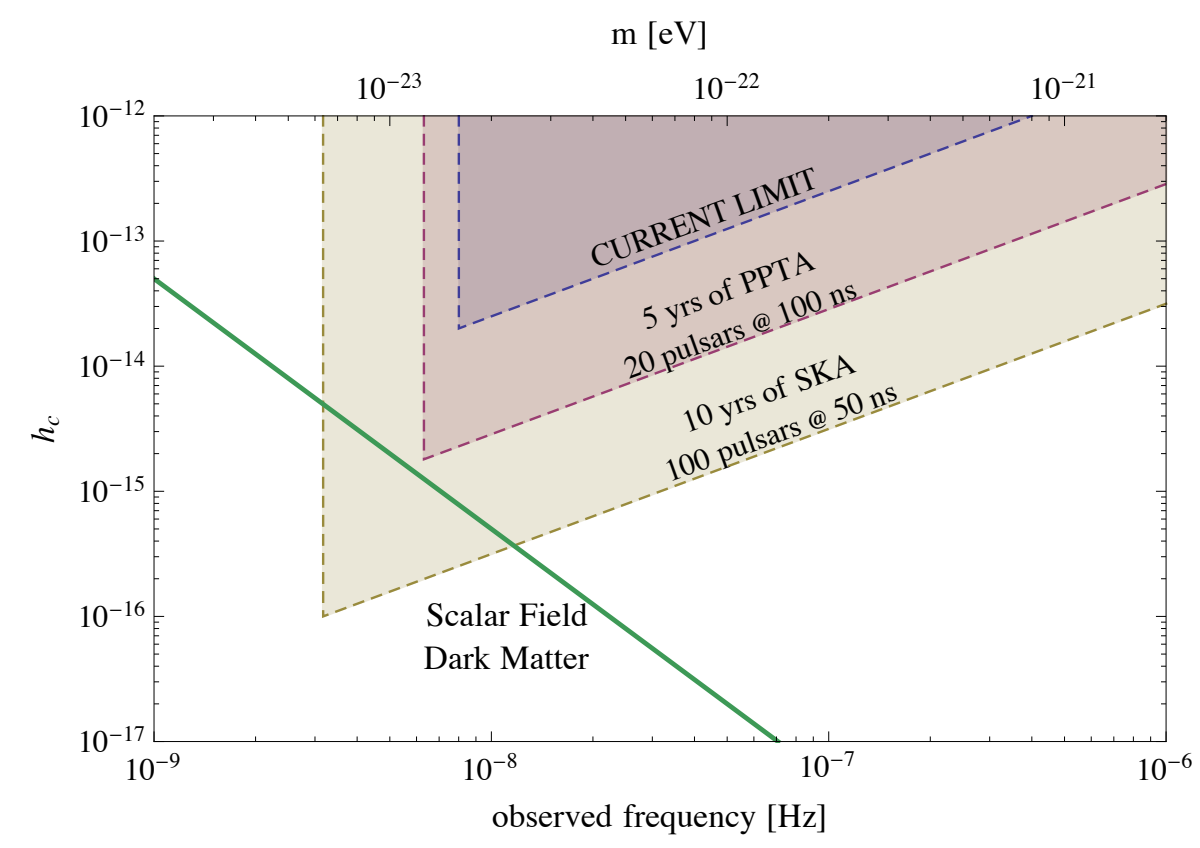

Figure 1: Pulsar timing signal from the scalar field dark matter (3.3) for a range of scalar field masses $m$. Shaded wedges represent the estimated sensitivity of various pulsar timing array observations (adopted from [7]). For masses below $10^{-23} \mathrm{eV}$ the scalar field behaves like hot dark matter, and is incompatible with the observed power spectrum of density perturbations $[3,11]$.

from stochastic gravitational wave background signal. First, the dark matter signal does not depend on the direction to the pulsar; second, it is monochromatic and would appear as an excess in the signal at a particular frequency related to the mass of the dark matter particle by Eq. (3.4).

\section{Acknowledgments}

The authors are indebted to Dmitry Gorbunov, Christof Wetterich, and Lāsma Alberte for helpful discussions. The research of AK is supported by Kreitman Foundation. VR has been supported in part by the grants RFBR 12-02-0653 and NS-5590.2012.2.

\section{References}

[1] J. R. Primack, "Triumphs and tribulations of Lambda CDM, the double dark theory," Annalen Phys. 524 (2012) 535; J. R. Primack, “Cosmology: small scale issues revisited,” New J. Phys. 11 (2009) 105029 [arXiv:0909.2247 [astro-ph.CO]].

[2] P. Bode, J. P. Ostriker and N. Turok, "Halo formation in warm dark matter models," Astrophys. J. 556 (2001) 93 [astro-ph/0010389].

[3] W. Hu, R. Barkana and A. Gruzinov, "Cold and fuzzy dark matter," Phys. Rev. Lett. 85 (2000) 1158 [astro-ph/0003365].

[4] J. Magana and T. Matos, "A brief Review of the Scalar Field Dark Matter model," J. Phys. Conf. Ser. 378 (2012) 012012 [arXiv:1201.6107 [astro-ph.CO]]; A. Suarez, V. H. Robles and T. Matos, "A 
Review on the Scalar Field/ Bose-Einstein Condensate Dark Matter Model,” arXiv:1302.0903 [astro-ph.CO].

[5] L. Amendola and R. Barbieri, "Dark matter from an ultra-light pseudo-Goldsone-boson," Phys. Lett. B 642 (2006) 192 [hep-ph/0509257].

[6] M. Kramer, 2, D. C. Backer, J. M. Cordes, T. J. W. Lazio, B. W. Stappers and S. Johnston, "Strong-field tests of gravity using pulsars and black holes," New Astron. Rev. 48 (2004) 993 [PoS MRU (2007) 020] [astro-ph/0409379].

[7] A. Sesana, A. Vecchio and C. N. Colacino, "The stochastic gravitational-wave background from massive black hole binary systems: implications for observations with Pulsar Timing Arrays," arXiv:0804.4476 [astro-ph]; A. Sesana and A. Vecchio, "Gravitational waves and pulsar timing: stochastic background, individual sources and parameter estimation,” Class. Quant. Grav. 27 (2010) 084016 [arXiv:1001.3161 [astro-ph.CO]].

[8] A. Khmelnitsky and V. Rubakov, "Pulsar timing signal from ultralight scalar dark matter," JCAP 1402 (2014) 019 [arXiv:1309.5888 [astro-ph.CO], arXiv:1309.5888].

[9] G. Hobbs, F. Jenet, K. J. Lee, J. P. W. Verbiest, D. Yardley, R. Manchester, A. Lommen and W. Coles et al., "TEMPO2, a new pulsar timing package. III: Gravitational wave simulation," arXiv:0901.0592 [astro-ph.SR].

[10] F. A. Jenet, G. B. Hobbs, W. van Straten, R. N. Manchester, M. Bailes, J. P. W. Verbiest,

R. T. Edwards and A. W. Hotan et al., "Upper bounds on the low-frequency stochastic gravitational wave background from pulsar timing observations: Current limits and future prospects," Astrophys. J. 653 (2006) 1571 [astro-ph/0609013].

"Constraining the coalescence rate of supermassive black-hole binaries using pulsar timing," Astrophys. J. 730 (2011) 29 [arXiv:1103.2808 [astro-ph.CO]].

[11] D. J. E. Marsh and P. G. Ferreira, "Ultra-Light Scalar Fields and the Growth of Structure in the Universe,” Phys. Rev. D 82 (2010) 103528 [arXiv:1009.3501 [hep-ph]]. 